\title{
CoviD-19 The impact of COVID-19 shielding on the wellbeing, mental health and treatment adherence of adults with cystic fibrosis
}

\author{
Authors: Kristina A Westcott, ${ }^{\mathrm{A}}$ Frances Wilkins, ${ }^{\mathrm{A}}$ Amelia Chancellor, ${ }^{\mathrm{B}}$ Alan Anderson, ${ }^{\mathrm{C}}$ Simon Doe, ${ }^{\mathrm{D}}$ \\ Carlos Echevarria ${ }^{\mathrm{D}}$ and Stephen Bourke $^{\mathrm{D}}$
}

People with cystic fibrosis (CF) were advised to undertake 'shielding' at home during the COVID-19 pandemic to reduce their risk of infection. We studied the impact shielding had on their wellbeing, mental health (GAD-7 and PHQ-9 scores) and adherence to treatment. 63 (46\%) of 137 people surveyed responded (19 anonymously; 44 gave their identity). Most (94\%) adhered to shielding advice 'all the time/often' but many (76\%) found this difficult with disruption of their routines, relationships and exercise habits. Treatment adherence rates were high and continued during COVID-19. Depression scores were low and remained stable. Clinically significant anxiety rates rose from $27 \%$ pre-COVID-19 to $54 \%$ during COVID- 19 and seven patients requested a psychology consultation from this study. There is a need to monitor the wellbeing of people with CF during the ongoing COVID-19 pandemic.

KEYWORDS: cystic fibrosis, COVID-19 shielding, anxiety, depression, adherence

DOI: 10.7861/fhj.2020-0205

\section{Introduction}

People with cystic fibrosis (CF) were considered to be extremely clinically vulnerable to coronavirus disease COVID-19 (SARS-CoV-2) and were advised on 23 March 2020 by the UK government to undertake protective 'shielding' (staying at home at all times and avoiding contact with people outside the household). ${ }^{1}$ From 1 August the guidance was relaxed to allow shielded individuals return to many activities with social distancing precautions. Shielding was an unprecedented intervention designed to protect vulnerable people from infection but little was known about any adverse effects it might have on their wellbeing and mental health. ${ }^{2}$ It is important to assess the impact of shielding in these patients in planning for managing the ongoing pandemic.

Authors: ${ }^{A}$ Clinical psychologist, Royal Victoria Infirmary, Newcastle upon Tyne, UK; ${ }^{B}$ assistant psychologist, Royal Victoria Infirmary,

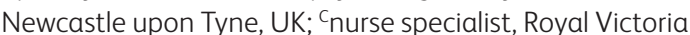

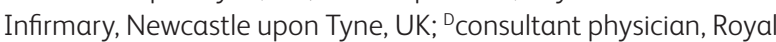
Victoria Infirmary, Newcastle upon Tyne, UK

\section{Methods}

At the end of July 2020, when shielding had been in place for 4 months, 137 adults with CF were sent an email inviting them to participate in an online survey of their experience of shielding. A reminder email was sent 1 week later. At the time 137 of 315 patients at our CF Centre were participating in the CF Health $\mathrm{Hub}(\mathrm{CFHH})$ project, which is a digital platform recording use of nebulised medication by e-track nebulisers, the data from which are used by the patient and clinical team to support adherence. ${ }^{3}$ These patients had consented to email communication and to associated research, with approval by our regional ethics committee and our institution's Caldicott guardian. Patients were given the option of replying anonymously or giving their identity confidentially to the researchers so that their responses could be linked to their clinical data.

The survey (supplementary material S1) first posed a series of questions:

1 What impact do you feel shielding has had on adherence to treatment, exercise, eating well, social support and relationships, sleep and daily routines, confidence, mental health and thoughts about their future?

2 Have you managed to adhere to the shielding advice provided by the government?

3 How difficult has it been for you to adhere to the shielding advice provided by the government?

4 What have you found most difficult about shielding?

5 How worried are you about shielding being lifted in the near future?

6 What worries you about shielding being lifted?

Responses were on 5-7-point scales, with questions 4 and 6 inviting free text comments. The respondents then completed the questions from the Generalised Anxiety Disorder assessment (GAD-7), which categorises anxiety as subclinical (scores $0-4$ ), mild (5-9), moderate (10-14) and severe (15-21), and the Patient Health Questionnaire (PHQ-9), which categorises depression as subclinical (0-4), mild (5-9), moderate (10-14), moderately severe (15-19) and severe (20-27). 4.5 For patients who gave their identity, GAD-7 and PHQ-9 scores were compared to baseline preCOVID-19 scores from their last annual review assessment, and their adherence with nebulised treatments was compared using $\mathrm{CFHH}$ data of their average adherence rate over the 4 months before and during shielding. For patients with data before and 
during COVID, changes were compared with the Wilcoxon rank test.

\section{Results}

Of 137 patients surveyed, 63 (46\%) responded: 19 (30\%) replied anonymously and $44(70 \%)$ gave their identity.

\section{Clinical characteristics}

For the 44 identified patients ( 25 men, 19 women), the mean age (range) was 32.7 (17-64) years, mean forced expiratory volume in one second $\left(\mathrm{FEV}_{1}\right)$ was $2.1(0.6-4.8) \mathrm{L}$, mean $\mathrm{FEV}_{1}$ percentage predicted was $57(16-108) \%$ and mean body mass index (BMI) was $22.8(16.4-28) \mathrm{kg} / \mathrm{m}^{2}$. They had chronic lung infections with Pseudomonas aeruginosa in 33, Staphylococcus aureus in six and Mycobacteroides abscessus in six patients. 33 (75\%) were on CF-modulator drugs (combinations of ivacaftor, tezacaftor and elexacaftor) and 11 (25\%) had CF-related diabetes. None developed COVID-19 infection.

\section{Survey responses}

For all 63 respondents, 59 (94\%) indicated that they adhered to shielding 'all of the time' ( $n=43)$, or 'often' $(n=16)$; four adhered 'sometimes', and none indicated 'rarely' or 'not at all' (supplementary material S2). 48 (76\%) found shielding difficult with a negative impact on exercise (65\%), social support (67\%), independence (29\%), sleep (70\%) and daily routines (57\%). Some $(40 \%)$ reported a positive impact on eating well; 28 (44\%) worried that when shielding was relaxed others might not adhere to social distancing (35\%) with subsequent risks of COVID-19 infection (43\%); $38 \%$ felt that shielding had no impact on adherence with treatments, $35 \%$ a positive impact and $27 \%$ a negative impact. Objective $\mathrm{CFHH}$ adherence rates were available for the 4 months of shielding for 42 (95\%) patients and showed a median of $86 \%$ (interquartile range $79-100 \%)$. For $26(60 \%)$ patients objective CFHH adherence rates for the 4 months before (median 90; IQR 71-99\%) and the 4 months during COVID-19 (median 86; IQR 79-100\%) were available, and showed no significant change with a median difference of 0 (IQR -4 to 8 ).

\section{Anxiety and depression}

Across all complete responses on the GAD-7 and PHQ-9 $(n=60)$, the median anxiety score was $5 / 21$ and the median depression score was 4/27; median pre-COVID anxiety scores were lower at 2/21, compared to during-COVID at 5/21. Median pre and during-COVID depression scores were the same (before and during COVID scores of 4). For the 44 identified respondents, comparable data before and during COVID were available for 41 (93\%) patients for GAD-7 and for $43(98 \%)$ for PHQ-9: there was a significant difference between the median anxiety scores before (2, IQR 0.5-6) and during (5, IQR 1-11) COVID ( $p=0.002$ ). Clinically significant ('mild' to 'severe') anxiety rose from $27 \%$ pre-COVID to $54 \%$ during COVID. There was no difference in median depressions scores before COVID (3, IQR 1-10) and during (3, IQR 2-12) COVID ( $p=0.09$ ) (Table 1). Seven patients requested a psychology consultation after completing this survey.

\section{Discussion}

This study provides novel data on the impact of shielding during the COVID-19 pandemic on adults with CF. These patients reported a high level of compliance with shielding although they found this difficult and disruptive to many aspects of their life. Depression levels were low and stable. Anxiety levels rose

\section{Table 1. Anxiety (GAD-7), depression (PHQ-9) and treatment adherence (CFHH) pre- and during the} COVID-19 pandemic

\begin{tabular}{|c|c|c|c|c|}
\hline Assessment & & Pre-COVID & During COVID & Difference \\
\hline \multirow[t]{6}{*}{ GAD-7 $(n=41)$} & Median [IQR] & $2[0.5-6]$ & $5[1-11]$ & $+3^{*}$ \\
\hline & Sub-clinical ( \%) & 73 & 46 & -27 \\
\hline & Mild (\%) & 7.3 & 27 & +20 \\
\hline & Moderate (\%) & 12 & 15 & +2.4 \\
\hline & Severe (\%) & 7.3 & 12 & +4.8 \\
\hline & 'Clinical’ (mild-severe; \%) & 27 & 54 & +27 \\
\hline \multirow[t]{7}{*}{ PHQ-9 $(n=43)$} & Median [IQR] & $3[1-10]$ & $3[2-12]$ & $0^{+}$ \\
\hline & Sub-Clinical (\%) & 53 & 53 & 0 \\
\hline & Mild (\%) & 21 & 16 & -4.7 \\
\hline & Moderate (\%) & 9.3 & 9.3 & 0 \\
\hline & Moderate-severe (\%) & 16 & 14 & -2.3 \\
\hline & Severe (\%) & 0 & 7 & +7 \\
\hline & 'Clinical' (mild-severe; \%) & 47 & 47 & 0 \\
\hline $\begin{array}{l}\text { CFHH Adherence }(n=28) \\
(\text { mean } \%)\end{array}$ & & $80 \%$ & $87 \%$ & $+7 \%^{+}$ \\
\hline
\end{tabular}


significantly but this may be seen as a normal response to an external threat and may have improved vigilance in managing the risk by promoting adherence to shielding and to treatment. None developed COVID-19 infection.

Early reports suggest that CF patients have had low rates of infection with COVID-19 and the course of the disease in CF has not been as severe as expected. ${ }^{6,7}$ This low incidence is likely to be due to people with CF adhering to shielding. These patients are already accustomed to hygiene precautions and segregation from other patients to reduce their risk of cross-infection with bacterial infections.

These CF patients generally coped well with the threat of COVID-19 and with shielding but had concerns about the potential risk of infection as shielding is relaxed, if others do not adhere to social distancing. The CF population is younger, with different sociodemographic features, than many other vulnerable groups with chronic lung diseases such as chronic obstructive pulmonary or fibrotic disease. Shielding had a disruptive effect on their independence, confidence, social relationships, education and employment. However, they may be better connected with their friends than other vulnerable groups by their use of technology and social media platforms. ${ }^{8}$ They have also had experience of developing resilience in the face of medical problems. Their coping strategies have been assessed in other studies using tools such as the CF-specific 'Ways of coping' scale which shows that 'optimistic acceptance' and 'hopefulness' are key coping strategies, with less reliance on 'distraction' or 'avoidance.'. Coping strategies help maintain wellbeing but also influence adherence with treatments. ${ }^{9-12}$ Several studies have shown that the symptoms of anxiety and depression are prevalent in particular subgroups of people with CF.13,14 These symptoms could be aggravated by the threat of COVID-19 and the isolation of shielding.

The strength of this study is in providing timely data using validated measures of anxiety, depression and treatment adherence. Limitations include the small sample size in a single centre study, possible biases from the population selected for the study and their response rate, and the limitations of survey questions in exploring all the issues involved.

Most patients adjusted well to the additional threat posed by COVID-19 and the stresses of shielding, but some required additional psychological support. Assessment of their wellbeing and mental health should form part of their standard clinical consultations and they should have access to specialist psychology services if needed.

\section{Supplementary material}

Additional supplementary material may be found in the online version of this article at www.rcpjournals.org/FH]:

S1 - Survey questionnaire

S2 - Survey responses

\section{References}

1 UK Government. Guidance on shielding and protecting people who are clinically extremely vulnerable from COVID-19. www.gov.uk/ government/publications/guidance-on-shielding-and-protectingextremely-vulnerable-persons-from-covid-19/guidance-on-shieldingand-protecting-extremely-vulnerable-persons-from-covid-19 [Accessed 13 April 2020].

2 National Institute for Health and Care Excellence. COVID-19 rapid guideline: cystic fibrosis. NICE guideline [NG170]. NICE, 2020. www.nice.org.uk/guidance/ng170.

3 National Institute for Health and Care Excellence. CFHealthHub for managing cystic fibrosis during the COVID-19 pandemic. Medtech innovation briefing [MIB219]. NICE, 2020. www.nice.org.uk/ guidance/mib219.

4 Spitzer R, Kroenke K, Williams JBW, lowe B. A brief measure for assessing generalized anxiety disorder: GAD-7. Arch Intern Med 2006;166:1092-7.

5 Kroenke K, Spitzer R, Williams JBW. The PHQ-9: validity of a brief depression severity measure. J Gen Intern Med 2001;16:606-13.

6 Cosgriff R, Ahern S, Bell S et al. A multinational report to characterise SARS-CoV-2 infection in people with cystic fibrosis. J Cyst Fibros 2020;19:355-8.

7 Colombo C, Burgel PR, Gartner S et al. Impact of COVID-19 on people with cystic fibrosis. Lancet Respir Med 2020;8:e35-e36.

8 Graetz BW, Shute RH, Sawyer MG. An Australian study of adolescents with cystic fibrosis: perceived supportive and nonsupportive behaviors from families and friends and psychological adjustments. J Adolesc Health 2000;26:64-9.

9 Abbott J, Dodd M, Gee L, Webb K. Ways of coping with cystic fibrosis: implications for treatment adherence. Disabil Rehabil 2001;23:315-24.

10 Askew K, Bamford J, Hudson N et al. Current characteristics, challenges and coping strategies of young people with cystic fibrosis as they transition to adulthood. Clin Med 2017;17:121-5.

11 Higham L, Ahmed S, Ahmed M. Hoping to live a 'normal' life whilst living with unpredictable health and fear of death: impact of cystic fibrosis on young adults. J Genet Counsel 2013;22:374-83.

12 Bucks RS, Hawkins K, Skinner TC et al. Adherence to treatment in adolescents with cystic fibrosis: the role of illness perceptions and treatment beliefs. J Pediatric Psychology 2009;34:893-902.

13 Quittner A, Goldbeck L, Abbott J et al. Prevalence of depression and anxiety in patients with cystic fibrosis and parent caregivers: results of the International Depression Epidemiological Study across nine countries. Thorax 2014:69:1090-7.

14 Goldbeck L, Fidika A, Herle M, Quittner A. Cochrane corner: psychological interventions for individuals with cystic fibrosis and their families. Thorax 2015;70:1089-91.

Address for correspondence: Dr Stephen J Bourke, Department of Respiratory Medicine, Royal Victoria Infirmary, Newcastle upon Tyne NE1 4LP, UK. Email: stephen.bourke1@nhs.net 